\title{
FRANCISCO MANZO ROBLEDO, Yo, Hernán Cortés: El Juicio de Residencia, EDITORIAL PLIEGOS, MADRID. 2013, 429 PÁGS.
}

\author{
José Antonio GUTIÉRREZ GTZ. \\ Claudio Antonio GRANADOS MACÍAS
}

Me ha sido enviado por el mismo autor este enjundioso e interesante libro de Francisco Manzo Robledo, que además de contar con el Doctorado en Ingeniería Civil por la Universidad de Guerrero, es Doctor de Literatura Latinoamericana en la Arizona State University y Catedrático de Literatura Colonial Latinoamericana de la Washington State University. El trabajo lo conocí parcialmente cuando llevaba a cabo la investigación en el Archivo General de Indias, porque departí su lectura en diversos momentos en una de mis estancias en dicho archivo. Aprovechando el espacio que nos brinda la Revista ....... Claudio y yo hemos querido reseñarlo para que los lectores conozcan el libro desde un punto de vista histórico-jurídico. Cabe resaltar que el autor echa mano de los acervos que guarda el Archivo General de Indias, de Sevilla, España; sobre todo el fondo "Justicia", así como de las "Cartas de Relación" y de otros documentos referentes al tema, como la obra de José Luis Martínez y documentos cortesianos por él publicados y otros.

El Dr. Manzo Robledo lo aborda desde la perspectiva de la literatura de la colonia en América Latina, "cuyo canon literario normalmente ha partido de documentos cuyo interés histórico ha rebasado el literario, estableciendo como documentos fundamentales productos de personajes que tienen qué ver con el llamado descubrimiento y conquista del continente" (pág. 9). El personaje central es Hernán Cortés, el más importante y controversial personaje en la conquista de México y que, como tal, su vida y documentos han sido sometidos por los historiadores al escrutinio desde diferentes puntos de vista; basta revisar las listas bibliográficas para encontrar variaciones. El tema en que se fija es "el juicio de residencia" que, para el autor, son pocos quienes lo han abordado por la dificultad para acudir al Archivo General de Indias.

Para nadie es ajeno que su memoria ha sido silenciada "a puntapiés durante cinco siglos y que aún descansa ignorado en los arcones de la historia de México, de esa misma nación de la que él puso los primeros cimientos" (pág. 11). Nacido en 1485 en Medellín, Extremadura, España, luego de hacer algunos estudios en la Universidad de Salamanca de latín y adquirir ciertos rudimentos legales, pasa a la isla Española en busca de fortuna en 1504; en 1511 el hijo del descubridor de América, Diego Colón, le encarga la conquista de Cuba, donde siendo alcalde de Santiago de Boracao se dedica a la cría de vacas, ovejas y yeguas, y a la extracción de oro, con lo que obtuvo alguna fortuna. En 1519 saldría como capitán general de la armada de la expedición que había preparado el gobernador Diego Velázquez para conquistar el

1. Departamentos de Historia y de Derecho de la UAA, e-mail: jagutier@correo. uaa.mex/claudiogranados72@ hotmail.com 
vasto imperio de México, del que había muestras de sus riquezas. La expedición saldría de las costas cubanas rumbo a Cozumel con once navíos, en febrero de 1519; bordando las costas llegó a Veracruz el Jueves Santo, 21 de abril de 1519, fundó la Villa de la Veracruz y erigió el primer ayuntamiento y cabildo en tierras aztecas. De Veracruz marchó a Tenochtitlán, a donde llega la mañana del 8 de noviembre, la que conquista después de varios eventos y de un largo asedio el 13 de agosto de 1521.

El tema central del libro es el juicio de residencia. Las acusaciones que habían llegado a España motivaron a Carlos V que ordenara elaborar el proyecto de juicio y que nombrara juez de residencia a Luis Ponce de León, quien llegó a ejecutarlo a la ciudad de México el 2 de julio de 1526. Lo que primero hizo fue suspender en sus funciones Hernán Cortés y despojarlo de la vara de gobernador; dos días después, por el pregonero Francisco González, hizo saber en la plaza pública y en otros lugares la real provisión que lo acreditaba juez de residencia y se anunció que, de dos a cuatro de la tarde oiría las quejas por 9o días. Ponce de León no tuvo tiempo de cumplir la orden real porque enfermó de gravedad y murió el 20 de dicho mes. Antes de morir había nombrado encargado del gobierno al licenciado Marcos de Aguilar, pero como éste moriría en marzo del año siguiente, quedó en suspenso la orden real u no se volvió a hablar del juicio

Dado el sesgo que había tomado el asunto, Cortés decidió viajar a España para presentar su caso al Emperador Carlos V y embarcó en diciembre de 1527. En el ínterin el emperador había erigido la Primer Audiencia formada por el presidencia Nuño Beltrán de Guzmán y los oidores Juan Ortiz de Matienza y Diego Delgadillo, la que tomó posesión del gobierno el 9 de diciembre de 1528. Como en abril Carlos V le había notificó que abriera el juicio, semanas después de tomar el gobierno elaboró un interrogatorio de 53 preguntas, 38 relativas a la situación general de Cortés y sus oficiales, en asuntos de gobierno, administración, fiscales y de justicia, más 15 acerca de la actuación de Cortés en cuestiones de piedad, moralidad, tiranía, infidelidad y apropiación del tesoro real y de las provincias de la tierra y dominio de los indios, y otras 15 sobre los oficiales reales.

Los primeros meses de 1529 se presentaron a declarar 90 testigos de cargo, la mayoría enemigos de Cortés. El 8 de mayo tenía la Audiencia liso el expediente sobre "Cargos que resultan contra Hernando Cortés"; pero como fueron tan burdos algunos de los testigos, el obispo Fr. Juan de Zumárraga notificó al Emperador que se trataba de un juicio amañado. El procurador, García de Llerena, presentó a la Audiencia el 12 de octubre de 1529 unos laboriosos "Descargos" en nombre de Cortés sobre las acusaciones que le fueron hechas en la "pesquisa secreta"; Cortés daría respuesta a los 11 principales cargos de la "instrucción secreta" el 14 de enero de 1534. En febrero de 1537 se notificó por cédula a Cortés que, como se hallaba inconcluso el juicio y enviados los documentos al Consejo de Indias, compareciera él o su procurador ante dicho Consejo para el seguimiento del proceso, a fin de escuchar la sentencia definitiva. En diciembre de 1539 Cortés embarcaría de nuevo a España para continuar su defensa, de donde ya no regresaría pues moriría el 2 de diciembre de 1547 a la edad de 62 años en Castilleja de la Cuesta, cerca de Sevilla, en casa de su amigo Alonso Rodríguez de Medina.

El tema del libro es el "juicio de residencia”. Unas palabras al respecto. El juicio de residencia fue un instrumento, una herramienta de control, que la corona española usó durante la colonia, más de una vez con excepciones, para ventilar el comportamiento de determinadas autoridades superiores durante su administración y mandato y lo llevaba a cabo al expirar su mandato. No eran un proceso judicial necesariamente punitivo, sino más bien una sana 
revisión a la que se sometía la actuación del cualquier oficial de la Corona al término de su mandato o en cualquier momento por causas graves. "Su propósito, dice José Luis Martínez, era el de regular el comportamiento de los funcionarios y permitir que oportunamente y en su propio lugar de residencia se ventilaran, sancionaran o aprobaran los actos de las autoridades. El juez al que se confiaba el juicio lo publicaba durante dos meses, a fin de que todos los que se consideraran agraviados pudieran intervenir. Habitualmente se presentaban interrogatorios previos a que los testigos de cargo y descargo debían contestar bajo juramento. Una vez concluidas las informaciones, se remitían al Consejo de Indias que dictabas sentencia y lo daba por terminado".

Pocos eran a quienes se les dispensaba. Hernán Cortes no fue una excepción. El primer intento de juicio se dio en 1526 al arribo a México de Luis Ponce de León, el que por muerte de éste y de su sucesor Marcos de Aguilar se detuvo hasta que Carlos V ordenó a la Primera Audiencia en abril de 1528 que lo efectuara. Durante el juicio Cortés y su equipo legal produjeron una serie de documentos de defensa contra los cargos propuestos, los que el autor toma como parte fundamental del trabajo. Cabe decir que lo que motivó al Emperador hacerle juicio de residencia fueron las acusaciones que llegaron a España contra Cortés. Poco después de la toma de Tenochtitlán comenzaron a llegar a España acusaciones contra Cortés por agravios a sus compañeros y por diversos actos cometidos durante la conquista, las que aumentaron en febrero de 1525 Rodrigo de Albornoz y Alonso de Estrada en la entrevista que tuvieron con Carlos V. Ante la cantidad de acusaciones, el emperador, primero pensó enviar a Nueva España al almirante Diego Colón, hijo del descubridor de América Cristóbal, para que le cortase la cabeza y castigase sus fechorías; pero la intervención de su padre Martín Cortés, de Fr. Pedro Melgarejo y del duque de Béjar logró que el emperador olvidara el proyecto y que mejor se le tomara residencia, lo que ya había acordado desde 1522. Así fue que nombró juez de residencia a Luis Ponce de León, pero que como dejamos dicho, por la muerte éste y del licenciad Marcos de Aguilar, que había quedado al frente del gobierno, el juicio quedó en suspenso hasta no tomar posesión la Primera Audiencia en 1528, cuando Cortés se encontraba en España arreglando sus problemas legales y buscando que se le anulara el juicio al no haber acusaciones formales todavía.

El presidente de la Primera Audiencia y sus oficiales abrieron el juicio a principios de 1528; para ello formuló un cuestionario sobre asuntos generales y sobre las "instrucciones secretas" o "capitulo secreto", para que las contestaran los testigos de cago; la lista de cargos fue fueron 101 en total. Se llamó a declara a 22 testigos, los que declararon del 23 de enero al 7 de abril. En mayo los procuradores de Cortés en México, Diego de Ocampo, Juan Altamirano y García de Llerena hicieron constar a la Audiencia lo irregular del juicio, porque todos los era notorios enemigos de Cortés, y en septiembre con firmes argumentos recusan a los tres jueces de la Audiencia, Nuño de Guzmán, Juan Ortiz de Matienza y Diego Delgadillo, presidente y oidores respectivamente; pero no la admitieron y continuaron el juicio e impusieron a Cortés una fianza de 9 a 90 mil maravedíes. En octubre García de Llerena presentó a la Audiencia a nombre de Cortés los "descargos" a las acusaciones y tachas presentadas por los testigos de cargo, documento que es una defensa de notable solidez jurídica de la actuación de Cortés. El expediente fue enviado al Consejo de Indias; en marzo de 1530 la reina envió una cédula a la Audiencia ordenándole que no se entrometiera más en el juicio de residencia y que todo lo remitiera al Consejo de Indias; es decir, por orden real quedaba sobreseído el

2 José Luis Martínez, Hernán Cortés, Fondo de Cultura Económica, México, 1997, págs. 463-464. 
juicio. La Segunda Audiencia quiso continuar el juicio, pero como había sido sobreseído, se suspendió.

Pero como en 1534 Cortés solcito al rey que se recibieran las declaraciones de sus testigos de descargo, éste ordenó que se abriera y prorrogara. Cortés con su equipo jurídico y algunos de sus capitanes y soldados preparó un interrogatorio al que debían responder los testigos de cargo; presentó para descargo un "interrogatorio" compuesto de 422 preguntas, unas fueron sobre asuntos generales referentes a su actuación pública, desde que su salida de Cuba hasta os días previos a la expedición del juicio, en 1526; otras se referían a cesuras personales generales y otras sobre las acusaciones la que aparecían en "las instrucciones secretas". Las secciones para escuchar y consignar las declaraciones de los testigos se extendieron del 21 de abril de 1534 al 27 de agosto del siguiente. El expediente se envió al Consejo de Indias. En 1537 Cortés recibió una cédula en que se le informaba que le juicio se hallaba inconcluso, por lo que concurriera él o su procurador ante dicho Consejo para dar seguimiento al proceso y escuchar la sentencia definitiva. Tres años después se presentó, pero al ver que no salía el fallo, en 1544 pidió al tribunal que declarar nulo el juicio, lo que nunca consiguió.

El autor relata los descargos en el capítulo III, de la pág. 83 a la 320. Tajante, una a una las refutó Cortés; sobre las que le hacen contra el servicio de Dios; por ejemplo la muerte a la muerte de su primera esposa, Catalina Suárez; contesta que asistía a misa diario, pero se acostaba con mujeres sin poner atención a la relación que pudiera haber entre ellas, con una madre o con una hija, no había mujer en su casa que despreciara; pero había que parecer cristiano; eso sí a las indígenas que escogían para su uso los conquistadores primero las bautizaban, luego lo demás (pág. 157). Y sobre las promiscuidades sexuales de que lo acusaron dice que no podían pasar porque era cristiano y "porque no están debidamente identificadas conforme al derecho las cópulas carnales que le achacan"; aparte sus acusadores "son hombres de baja suerte e manera, e infames".

Resumamos ahora el libro del Dr. Manzo Robledo. En el capítulo primero el autor nos introduce en el contexto en que fundamentar su trabajo. Nos dice que responde al cuestionamiento siguiente: ¿Cómo fue posible que Hernán Cortés salvara su vida, lograra el otorgamiento del título de "Marqués del Valle de Oaxaca" y recibiera 23,00o indios para su servicio, a pesar de los cargos en su contras? En el primero de los capítulos plantea sucintamente la crítica literaria del juicio de residencia y señala tres conceptos: 1) La realidad relativa que se utiliza para discutir los documentos resultantes de la defensa en el segundo procedimiento. 2). La construcción del acto, espacio individual y social, donde Cortés se legitima, y 3) El acrecentamiento del acto por medio del proceso de escritura, donde el conquistador reivindica su derecho a compartir el poder con el monarca, al que le manifiesta su fidelidad, bajo una línea de jerarquía que traza entre Dios, el Rey y el concepto del Yo.

El realismo planteado, dice, es definido en la literatura como referencia al objeto temático y la técnica por la cual un trabajo literario ha sido creado. El realista, tratando de presentar su vida permite que la historia se cuente sola, por verdadera. El realismo relativo es utilizado junto con el concepto de acto, espacio social que incluye a todos los existentes -simplemente es el espacio de acción de cada cual, con un acto-. La aplicación del realismo relativo en la obra, aparece como manifestación de lo que debemos entender partiendo, de que un mismo evento puede ser interpretado de diferentes maneras por observadores imbuidos y en sí mismos bajo diferente entorno inmediato (acto-espacio). Manifiesta, así, que el episodio dala fundación del Cabildo de la Vera Cruz oficialmente propone a Hernán Cortés como el representante "legal" de la Corona y de Dios en la nueva tierra; su defensor García de Llerena 
escribe a nombre de Cortés en descargo de su ilegalidad: "Y porque el dicho don Hernando Cortés vio, que de poblarse esta tierra, Dios Nuestro Señor sería muy servido, y Vuestra Majestad por el consiguiente, y vuestros fisco y rentas muy aumentados, la quiso poblar en nombre de Vuestra Majestad..." (pág. 31).

En el capítulo segundo menciona la documentación en torno al juicio, el que se implanta por la necesidad de organizar la Real Hacienda en las nuevas posesiones y para que la Corona pudiera recolectar el quinto real los impuestos y derechos de los permisos otorgados, y para controlar las tierras y riqueza que en nombre de los soberanos españoles tomaban los conquistados. "El juicio, dice, contiene instrumentos de revisión y control, normalmente se llevaba al término del mandato de una autoridad -gobernadores, virreyes y otras-“; en el caso de Cortés, los documentos que forman la base del estudio se corresponden a los hechos acontecidos a partir del 4 de julio de 1526 en que arriba a la ciudad de México de Luis Ponce de León, el juez instructor e investigador de la causa nombrado por el Rey, quien le retira la vara y le a ordena dejar el gobierno de Nueva España en sus manos. Y Como después de anunciar por pregón del juicio de residencia, cae Ponce de León gravemente enfermo y muere el 20 de julio, deja el gobierno en manos de Marcos de Aguilar; pero como también muere al año siguiente, lo deja en manos del tesorero real Alonso de Estrada, el cual por desacuerdos con Cortés lo expulsa de la ciudad de México; éste marcha a España en marzo de 1528, para pedir al Rey la anulación de su juicio, ya que no había acusaciones en su contra.

En el ínterin que Cortés se reunía con el rey, en la ciudad de México se instala la Primera Audiencia; el presidente Nuño de Guzmán, cumpliendo la real orden de abril 5 de 1528, inicia, en ausencia de Cortés y sus oficiales. La Audiencia formula un cuestionario dirigido a los testigos de cargo, el que responden entre el 23 de enero y 7 de abril del 1529. Produce un gran número de documentos: declaraciones de testigos, recusaciones, cargos, descargos, cuestionarios de la parte acusadora y de la parte acusada. Respecto a los cargos contra Cortés, el autor maneja dos listados generales, el de "cargos menores" que era una recopilación de las acusaciones en su contra, y el de cargos mayores, que son el resultado de las declaraciones efectuadas por los 22 testigos cuestionados, los que se acumulan con los llamados cargos en la "pesquisa secreta".

Agrega el autor que, sobreseído el juico, en octubre de 1533 Cortés y su equipo legal lograron del rey que se reabriera el juicio y admitiera la documentación de su defensa. En ella incluyen, en la que incluyen 380 preguntas dirigidas a los testigos de descargo para oponer las excepciones a las imputaciones hechas en el procedimiento de la Primera Audiencia, más 42 para atajar la acusación de la "pesquisa secreta". El autor analiza la documentación a través de cinco puntos a observar: 1. La carta remitida por Cortés a España, es una petitoria al Rey y de los vasallos firmantes para legitimar su condición en el territorio de lo que sería Nueva España; 2. Los vasallos convalidan la legitimación del grupo al otorgar la calidad de Capitán General y Justicia Mayor que el cabildo de la Vera Cruz confirió a Cortés, lo que en su conjunto, a través de la figura de la constitución y autogobierno de los ayuntamientos castellanos, les permitió sostener el nombramiento de Cortés y la calidad legal de los expedicionarios hasta en tanto el Rey confirmara esa calidad; 3 . El informe de Cortés al rey resalta su figura, lo que iniciaría el recelo del Rey y su Consejo hacia el conquistador; 4. La carta es también una queja contra Diego Velázquez y Pánfilo de Narváez, y 5. La petición refuerza 
la solicitud de convalidación del nombramiento de Cortés por los servicios prestados a la corona.

En el capítulo tercero, el más extenso casi 200 páginas, el autor analiza la listas general de cargos desde el realismo, relativo la contraposición entre los partidos en pugna -la visión del grupo encabezado por Nuño de Guzmán, los de la Primera Audiencia y los enemigos de Cortés, que deseaban el poder y las ganancias que Nueva España producía, anhelos sostenidos por el poder oficial que les concedió Carlos V-, y el bando del propio Cortés auxiliado por su equipo legal y los testigos de descargo afines a Cortés o compañeros, que querían conservar el poder y que sería la causa de establecer como cargos medulares en el juicio. Cortés buscaba mantener el poder absoluto que la conquista le otorgaba según los cánones del derecho medieval, ya que la lealtad hacia el Rey se mostraba al compartir el quinto que el propio monarca imponía sobre las riquezas ganadas en batalla, al contribuir al tesoro real con los impuestos, derechos y aprovechamientos que debían tributarse a la Real Hacienda, los que iban de la mano de los permisos y autorizaciones.

Dice el autor que el lenguaje que utiliza Cortés utiliza en el proceso de descarga va dirigido por estrategia legal a Carlos $\mathrm{V}$ y al Consejo de Indias que conoce del procedimiento, no a la autoridad acusadora que es la Audiencia que presenta los cargos; así Cortés permanece dentro de una Dios-Rey-Yo (pág. 88), lo que sugiere un acomodo conveniente desde el punto de vista de Cortés. La conciencia la arregla con Dios a través de sus representantes terrenos los frailes y el Papa-; mientras que los hechos con cuestionamiento de legalidad, los justifica ante el Rey, a quien tanto ha aportado. La situación de Cortés es complicada porque va la vida de por medio, debido a que desobedece a la autoridad representante del Rey. Esta tesitura es la que le obliga a defender su vida, sus bienes y su futuro; de ahí que en el documento de descargos emplee una visión de conquista y que conteste a los cargos con argumentos justificatorios, legaloides o simplemente negando o ignorando el cargo. Arremete contra sus acusadores y enemigos a los que trata de desacreditar en una época donde el estatus lo es todo. Elabora, entonces, los relatos laterales, juicios de valor y carácter que la defensa adjudica a los personajes y los tacha de testigos falsos.

Cortés promueve una especie de metaotredad: en lugar de una segmentación simple de "los españoles" -los conquistadores- y los "otros" -los conquistados-, reinventa la segmentación. Establece su actuación en nombre de Dios y el papel de los otros españoles como un grupo reducido, luego establece en la jerarquía a los indígenas libres y finalmente los esclavos: un sistema de círculos concéntricos en el que Cortés pretende contener a los demás, realidad relativa de la metaotredad. Se advierte que en los cargos se impone un orden que no es estrictamente cronológico, los que inician con el primer acto del trueque de Cortés como jefe de expedición que es cuestionado por Velázquez, a Capitán General y Justicia Mayor en las nuevas posesiones, en servicio de Dios y el Rey. Para los descargos el documento es formulado a partir de un ataque ya definido, en donde los puntos débiles/fuertes de la parte acusadora los precisa, y desarrolla los hechos contradiciendo de la mejor manera posible las propuestas del cargo.

Para la crítica el autor maneja dos apartados, uno que contiene los cargos contra el servicio del Rey y y el otro los cargos contra el servicio de Dios. En el primero enlista los siguiente cargos: 1. Problemas con el quinto real; 2. Mal trato a los indígenas; 3. Nepotismo; 4. Movimiento independentista de Cortés; 5. La mala aplicación de la ley, y 6. la mala administración de la tierra y contra el bien común; éstos cuestionamientos de legalidad los justifica ante el Rey "a quien tanto ha traído bajo su dominio". En cuanto a los cargos contras el servicio de 
Dios señala que sólo son 8 los establecidos; concretamente que permitiera y solapara el juego de azar en su gente, haber dado muerte su primera esposa, Catalina Suarez y excesos sexuales; los testigos y él los rechaza, "porque asistía a misa diario". Y referencia a que se acostara con mujeres en su casa sin poner atención a la relación que pudiera haber entre ellas, pues no había mujer en su casa que despreciara; declararon los testigos: "La conciencia la arregla con Dios por medio de sus representantes en la tierra: los curas y su representante máximo el Papa” (pág. 94).

Concluyendo, los documentos de "descargos" y los "cuestionarios" representan a Cortés como la figura de un caballero legendario que lleva a cabo la conquista y establece la dominación de nuevas posiciones para su Rey; se le recrea como un nuevo Rodrigo Díaz de Vivar, como un caballero que obedece a la dos majestades: al Rey y Dios; también manifiestan que el Rey debería sentirse en deuda con un vasallo que tanta riqueza trajo a sus dominios. En todo momento en los documentos de defensa se busca la absolución de los cargos, en cuanto que lo que el capitán general anhelaba era la autoridad y permiso real para continuar con los descubrimientos, más poder y riqueza y, sobre todo, fama, y un gran número de almas para Dios y el bien de la real conciencia.

La narración que percibimos en el libro del Dr. Manzo Robledo es lineal como lo sería el formato del cuestionario, y respeta la cronología de los eventos históricos señalados, los que estructura de la siguiente manera: 1-. Introducción, en donde se repasan los eventos importantes con respecto a las expediciones iniciales al continente. 2-. La etapa preparatoria, en donde incluye todas las peripecias que requirió Cortés para desembarazarse de la custodia de Velázquez, lo que manifiesta a un Cortés conquistador y conocedor de las encrucijadas de la legalidad, ya que antes de hacerse a la mar solicita al contador real que dé fe de los navíos que salen desde Cuba en la empresa, preparando lo que venía. 3-. La etapa de preconquista, donde narra el arribo a Cozumel, su encuentro con los indígenas a los que sometió al imperio de Dios y el Rey. 4-. La llegada al continente y sus primeras escaramuzas con los indígenas, donde hace patente sus dotes militares. 5-. La ruptura definitiva con Velázquez para cambiar los objetivos de la expedición y patentizar su posición ante la Corona; reconoce al Rey como su inmediato superior y, para ello, funda la Villa Rica de la Veracruz y designa autoridades en nombre del Rey, las que lo eligen Justicia Mayor y Capitán General. 6-. La actuación anticipada a los designios reales, momentos en que procede en nombre de Dios y el Rey y envía regalos de oro y joyas, y le da cuenta de la nueva tierra y sus pobladores que ha sometido al poder del trono español. 7-. La postconquista, en donde en ocasiones rompe con la cronología y regresa a hechos pasados, aparentemente buscando justificaciones a una acusación que no existe en el escrito, pero que se infiere del contexto que produce el documento del proceso inicial en su conjunto. 8-. Las tachas, donde se desacreditan a los enemigos de Cortés con una opinión contraria a algunos personajes declarados sus contrarios. Finalmente se hacen algunas menciones respecto a que Cortés era reconocido verdadero conquistador y héroe por muchos en España y Europa; por ello, la conquista es para Cortés un ropón protector que nadie le podía arrebatar, una empresa producto de su genio militar, estratégico y político que nadie podía negar.

$\mathrm{El}$ autor concluye observando que en los documentos suscritos no hay reflejo de temor alguno a la veracidad de los asuntos tratados en torno al juicio de residencia, en virtud de su protección ganada como Conquistador de Nueva España, como también que de muchas formas había manifestado lealtad al Rey y le había generado múltiples riquezas. Sin embargo, reiteramos que nunca existió voluntad para desahogar el juicio de residencia, para que Cor- 
tés pudiera gozar plenamente de los beneficios de la conquista. Como nunca fue absuelto, no quiso abandonar España. Cortés fue encerrado en un limbo legal. Agregamos, ¿la dilación fue producto de la experiencia que la Corona adquirió con el caso de Cristóbal Colón?, ¿o fue porque temía Rey que se independizara y formar un reino aparte? Que Cortés fuera reivindicado como Conquistador y Capitán General por el emperador y que le permitiera pasar de la virtual ilegalidad a la máxima autoridad de la tierra conquistada, pudo ser a que quería legitimar la conquista para evitar los cuestionamientos de los países enemigos de España. 